\title{
BIOFLUX nach Tödt
}

\section{Apparatur zur einfachen und schnellen elektrochemischen Sauerstoffmessung}

\section{Meßprinzip}

Das Prinzip der elektrochemischen Sauerstoffmessung beruht auf dem Befund, daß die Stromdichte geeignet zusammengestellter galvanischer Elemente unter bestimmten Versuchsbedingungen als exaktes $\mathrm{Ma}$ für den im Elektrolyten gelösten Sauerstoff verwendet werden kann. Gegenüber der chemischen Sauerstoffbestimmung (z. B.

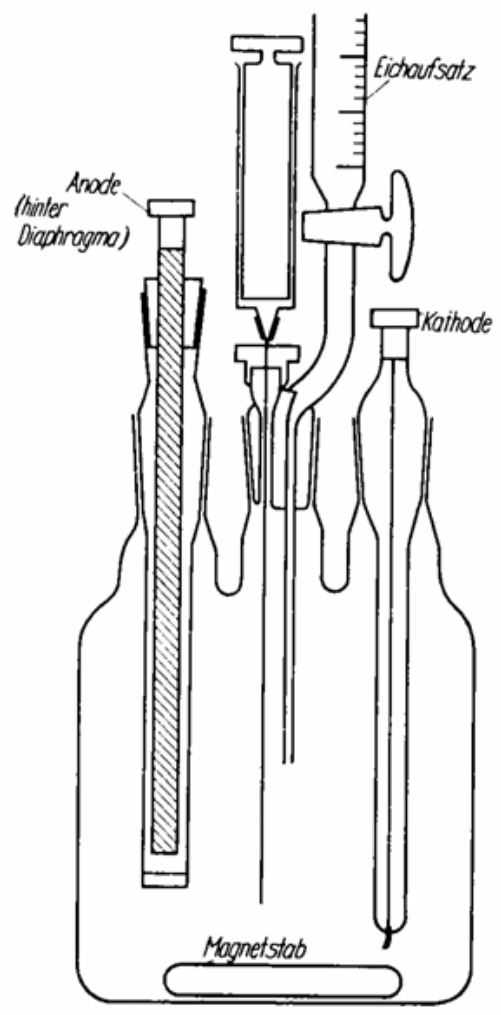

Abbildung A.

Schematische Darstellung des Bioflux nach Winkler) sind die Einfachheit und Schnelligkeit (sofortiges Ablesen oder automatische Registrierung der Stromstärke) sowie die zeitlich lückenlose Erfassung von Sauerstoffkonzentrationsänderungen bemerkenswert. Das Verfahren hat sich daher in den letzten Jahren wachsend neue teils technisch, teils wissenschaftlich wichtige Anwendungsgebiete erobert.

Durch Verwendung von Goldamalgamelektroden als Meßelektroden, welche eine hohe Überspan. nung haben, kann man in Kombination mit einer Zinkelektrode bei $\mathrm{p}_{H}$-Werten von 6 bis 7 oder mehr messen, ohne zusätzliche Ströme durch direkte Wasserstoffentwicklung befürchten zu müssen. Zwischen $p_{H} 6$ bis 7 und etwa 3,5 ist in Verbindung mit Goldamalgam Eisen und unterhalb $\mathrm{P}_{\mathrm{H}}$ 3,5 Blei als Anode brauchbar. Die Anoden werden meist durch ein Diaphragma von der Meßlösung getrennt und meist in einer gesättigten $\mathrm{KCl}$ - oder $\mathrm{NaCl}$-Lösung untergebracht. Für Blei wird vorzugsweise Standardacetat als Elektrolyt im Anodenraum verwendet. Die Anoden sollen möglichst so ausgebildet sein, daß sie bis dicht an das Diaphragma heranreichen.

$\mathrm{Da}$ es sich bei der elektrochemischen Sauerstoffmessung um die Bestimmung von Diffusions. strömen (Grenz- bzw. Restströme) handelt, muß zurErzielung quantitativer Ergebnisse eine gleich. mäßige Flüssigkeitsbewegung vorhanden sein. Dies wird entweder erreicht durch gleichmäßige Durchströmung (Durchflußgefäß)oder durch eine 
in Abbildung A schematisch dargestellte Magnetrührung in einem von der Luft abgeschlossenen Meßgefäß, in welches durch Glasschliffe die eigentliche Meßelektrode (edle Elektrode, Kathode) und die Gegenelektrode (unedle Elektrode, Anode) sowie die zu messenden Lösungen eingefüllt werden.

\section{Beschreibung der Apparatur}

In der Abbildung B ist die meßfertige Apparatur ohne Thermostaten (Klein-Bioflux) dargestellt. Das Meßgefäß wird in die auf den Magnetrührer aufgesetzte Kunststoffwanne eingesetzt. In der Mitte des Bodens der Kunststoffwanne befinden sich $3 \mathrm{Gummi-}$ zylinder zur Halterung des Meßgefäßes, welches in $2 \mathrm{Größen} \mathrm{mit} 50$ und $100 \mathrm{ml}$ Inhalt vorgesehen ist. Außer den beiden Einführungsschliffen für die beiden Elektroden sind am Meßgefäß noch 2 weitere Schliffe vorhanden. In den einen dieser Schliffe wird die Eichbürette, deren Wirkungsweise auf Seite XVII beschrieben ist, eingesetzt. Der 4. Schliff dient zur Aufnahme eines Gaseinleitungsrohres oder Thermometers.

Die Kunststoffwanne enthält noch für den Fall, daß ein Thermostat angeschlossen werden soll, ein Zufluß- und ein Abflußrohr. Der Wasserabfluß kann in 2 verschiedenen Höhen eingestellt werden, je nach Verwendung des 50 oder $100 \mathrm{ml}$ enthaltenden Meßgefäßes. Die Thermostatisierung kann auch nach Abbildung $C$ durch ein Kontaktthermometer und einen Heizwiderstand im Meßgefäß selbst stattfinden. Das zur Messung der Stromstärke dienende Meßinstrument ist wasserdicht gekapselt und enthält 8 Stromstärkemeßbereiche von 6 bis 1200 Mikroampere. Ferner ist ein Schalter für Kurzschluß angebracht.

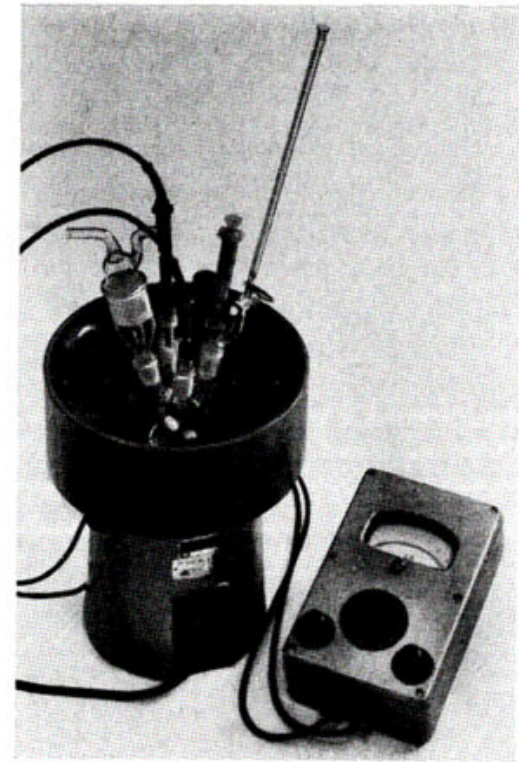

Abbildung B. Klein-Bioflux

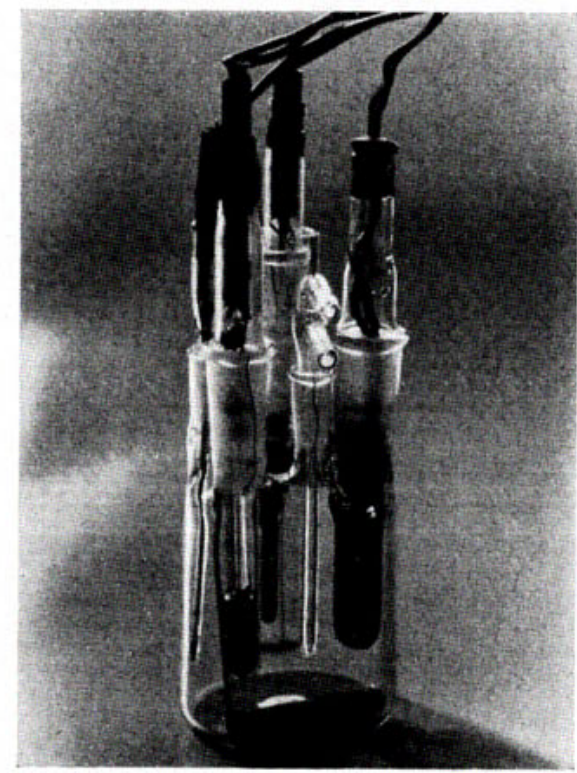

Abbildung C.Meßgefäß mit Thermostatisierung 
Die Abbildung $D$ zeigt eine Apparatur mit eingebautem Thermostaten und Magnetrührer (Groß-Bioflux). Elektrodengefäß, Kunststoffwanne und Meßinstrument entsprechen dem Klein-Bioflux. Unten an der Stirnplatte sind die Schalter zum Einschalten des Lichtnetzes, des Thermostaten und des Magnetrührers angebracht.

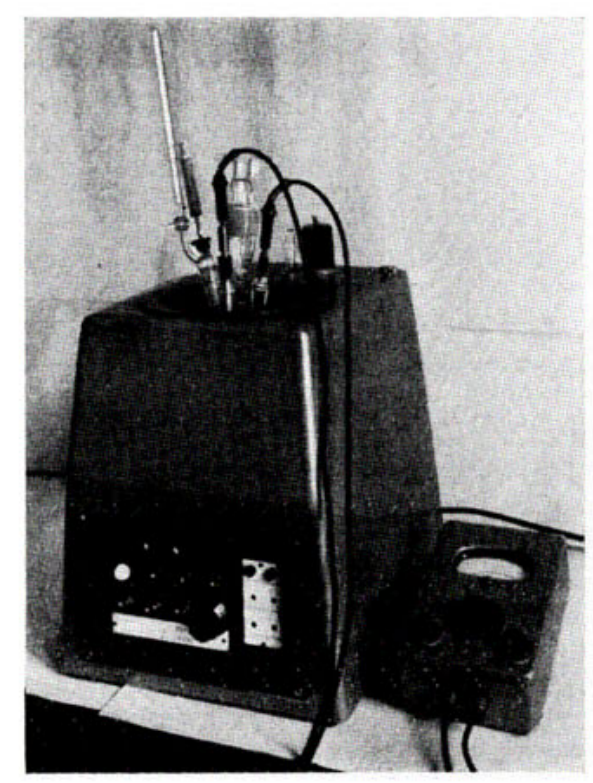

Abbildung D. Groß-Bioflux

3. Inbetriebnahme

a) Klein-Bioflux: Einlegen des Rührstäbchens in das Meßgefäß, Füllen des Meßgefäßes, Einsetzen des Meßgefäßes zwischen die 3 Gummizylinder, Anschließen des Meßinstrumentes an die Elektroden, Einschalten des Magnetrührers. Das Meßinstrument zeigt jetzt den gelösten Sauerstoff an. Es ist zweckmäßig, den Anodenraum einige Zeit vor der Messung mit gesättigter Kaliumchlorid- oder Natriumchloridlösung zu füllen, da eine gewisse Zeit vergehen kann, bis die Lösung das Diaphragma durchdringt und da bis zu diesem Zeitpunkt unter Umständen ein zu hoher innerer Widerstand zu niedrige Anzeigen hervorrufen kann.

b) Groß-Bioflux: Ein Unterschied zum Klein-Bioflux besteht nur darin, daß vorher das Kontaktthermometer auf die gewünschte Temperatur einzustellen ist und $\mathrm{da} B$ zur Inbetriebnahme die Schalter auf der Stirnplatte einzuschalten sind. Zum Anheizen des Thermostaten wird die Heizung auf 500 Watt und für den Betrieb auf 100 Watt geschaltet.

\section{Eichung}

Am Beginn jeder Messung ergibt sich automatisch ein Wert, welcher der bei Luftsättigung vorhandenen Sauerstoffkonzentration entspricht. Damit ist aber bereits die Beziehung zwischen abgelesener Stromstärke und Sauerstoffkonzentration, also auch die Eichkurve, gegeben. Jetzt braucht nur noch die Elektrodengröße so gewählt zu 
werden, daß die Eichkurve linear verläuft, was ohne Schwierigkeiten möglich ist. Es genügt also dann ein Punkt, der Luftsättigungswert, für die Eichung. Der Luftsättigungswert kann stets als exakte Meßgrundlage (oder Vergleichsgröße) benutzt werden. Sollten sich unter ungünstigen Meßbedingungen absinkende Stromwerte zeigen, die ihre Ursache in wenig übersichtlichen Konzentrationsverschiebungen innerhalb der Diffusionsschicht und an der Elektrodenoberfläche haben, wird eine einwandfreie Bestimmung des Luftsättigungswertes dadurch erreicht, daß der Strom nur so lange eingeschaltet bleibt, bis der angezeigte Wert nicht weiter steigt. Dies ist nach 10 bis 20 Sekunden der Fall. Wegen der starken Abhängigkeit der Diffusionsgeschwindigkeit von der jeweiligen Stellung der Elektroden und weiterer den Diffusionsweg beeinflussenden Faktoren muß der Luftsättigungswert bei jeder Versuchsreihe verschieden sein. Dies berührt jedoch die Genauigkeit der Ergebnisse nicht, da diese auf die unveränderliche Löslichkeit des Sauerstoffs, also eine Naturkonstante, bezogen werden, wie die im nächsten Abschnitt 5 durchgeführte Berechnung zeigt. Bei besonders hohen Anforderungen an die Genauigkeit ist die Luftsättigung auf $760 \mathrm{~mm}$ Normaldruck bezogen worden.

Eine weitere Eichung ist jederzeit dadurch mögiich, daß man durch Gaseinleiten (z. B. Stickstoff) den Sauerstoff weitgehend austreibt (was in 1 bis 2 Minuten mit Hilfe des Schliffaufsatzes mit Einleitungsrohr erreicht wird) und jetzt bekannte Sauerstoffmengen hinzugibt. Dies geschieht, indem man mit Hilfe der auf dem Meßgefäß in einem Schliff angebrachten graduierten Bürette eine bekannte Anzahl von ml luftgesättigter Lösung zugibt. Dies erfolgt durch eine Injektionsspritze. Die zugefügte Menge wird an der graduierten Bürette abgelesen. Damit hat man die theoretisch zugesetzte Sauerstoffmenge und liest die dazugehörige Stromstärkeerhöhung ab. Eine solche Zugabeeichung ist in weniger als einer Minute durchführbar und gestattet jederzeit, in dem jeweiligen Meßbereich sich von der Richtigkeit der Eichung zu überzeugen. Die nachstehende Tabelle enthält Luftsättigungswerte bei verschiedenen Temperaturen und Salzgehalten.Wird mit anderen Lösungen gearbeitet, so kann der Luftsättigungswert mit der üblichen Sauerstoffmessung nach Winkler festgestellt werden.

Luftsättigungswerte in $\mathrm{mg} /$ Sauerstoff im Liter Lösung

\begin{tabular}{c|c|c|c|c|c}
$\mathrm{t}$ & $\mathrm{H}_{2} \mathrm{O}$ & $0,1 \mathrm{n}$ & $1 \mathrm{n} \mathrm{KCl}$ & $1 \mathrm{n} \mathrm{Na} \mathrm{SO}_{4}$ & $1 \%$ Phosphatisg. \\
\hline 20 & 9,1 & 8,6 & 6,9 & 6,3 & 8,4 \\
30 & 7,5 & 7,1 & 6,0 & 5,45 & 7,0 \\
40 & 6,4 & 6,1 & 5,2 & 4,8 & 6,1 \\
50 & 5,5 & 5,2 & 4,6 & 4,2 & 5,2
\end{tabular}

5. Bisherige Ergebnisse und Berechnung der $Q_{0_{2}}-$ Werte

Die offenbar größte und wichtigste Anwendungsmöglichkeit der elektrochemischen Sauerstoffmessung besteht in der Messung des Sauerstoffverbrauchs durch die Atmung von Mikroorganismen. Alle Einflüsse, welche die Atmung herabsetzen oder beschleunigen, sind auf diese Weise meßbar[7] (Messung der Gifteinwirkung, Abbildung $E$ sowie der Atmungsbeschleunigung [7] Abbildung F). Nach Wa rbu rg wird die Atmung als $\mathrm{QO}_{2}$-Wert angegeben $\left(\mathrm{mm}^{3}\right.$ verbrauchter Sauerstoff pro Stunde und $\mathrm{mg}$ Trockensubstanz). Die Umrechnung der am Stromanzeigeinstrument abgelesenen Werte in $\mathrm{QO}_{2}$ kann folgendermaßen vorgenommen werden: Durch die Ablesungen erhält man zunächst den Stromabfall in Mikroampere/Minute. Die bei Luftsättigung und $20^{\circ} \mathrm{C} \mathrm{im} \mathrm{Liter} \mathrm{gelöste} \mathrm{Sauerstoffmenge} \mathrm{von} 8400 \mu \mathrm{g}$ (nach der Tabelle in $1 \%$ igem 


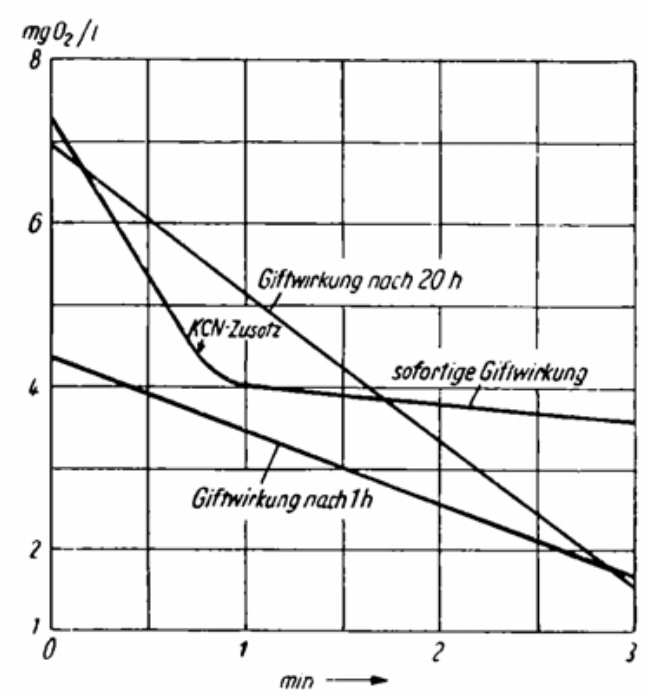

Abbildung E. Vergiftung von Hefe durch $\mathrm{KCN}$. $1 \% \%$ Hefe $+10_{0}^{0}$ Glukose, $5 \cdot 10^{-4}$ molar KCN

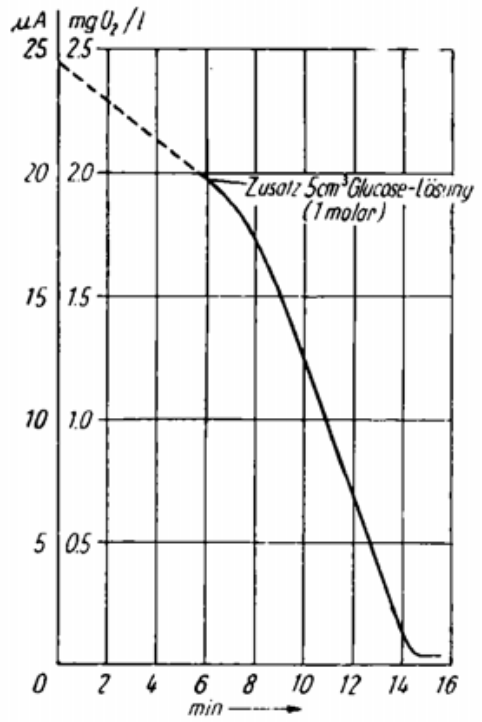

Abbildung F. Atmungsbeschleunigung der Grünalge Chlorella durch GlukoseZusatz

Abbildung G. Photosynthese durch Elektronenblitze von $2 \cdot 10^{-9} \mathrm{sec}$ :

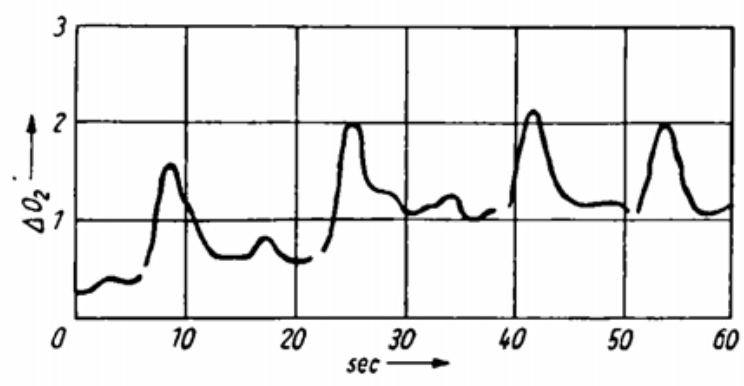

Phosphatpuffer) liefert, durch die bei Luftsättigung abgelesenen Mikroampere dividiert, den Sauerstoffgehalt pro Mikroampere Stromstärkeänderung, bezogen a sf einen Liter. Dieser ergibt mit den abgelesenen Mikroampere pro Minute multipliziert die Sauerstoffänderung in $\mu \mathrm{g}$ Sauerstoff pro Minute und Liter. Um den $\mathrm{QO}_{2} \mathrm{zu}$ erhalten, muß man jetzt noch mit 60 (Umrechnung von Minuten in Stunden) urd 0,7 (Umrechnung von $\mu \mathrm{g}$ in $\mathrm{mm}^{3}$ ) multiplizieren und durch das Trockengewicht $\mathrm{n}$ $\mathrm{mg}$ pro Liter dividieren. Die gesamte Ausrechnung läßt sich durch die folgende For $m \geqslant l$ ausdrücken:

Abgelesene Mikroampere pro Minute $\cdot 8400 \cdot 60 \cdot 0,7$

Mikroampere bei Luftsättigung - Trockengewicht in $\mathrm{mg}$ Liter

Sämtliche Größen außer den abgelesenen Mikroampere pro Minute sind bei einer Versuchsreihe gleich, so daß sich für jede Versuchsreihe ein einfacher Faktor ergitt, mit welchem die abgelesenen Mikroampere pro Minute zu multiplizieren sind, um den 
$\mathrm{Q}_{\mathrm{O}_{2}}$-Wert zu erhalten. Die zu messenden Organismenkulturen können, wie bei der Eichung beschrieben, mit der Injektionsspritze quantitativ zugegeben werden.

Auch die Photosynthese ließ sich in sehr einfacher Weise elektrochemisch messen. So konnte auf diesem Wege die durch die Grünalge Chlorella photosynthetisch entstandene Sauerstoffmenge bereits im Laufe weniger Sekunden durch ein schreibendes Galvanometer quantitativ registriert werden [7] (Abb.G). Die Empfindlichkeit im

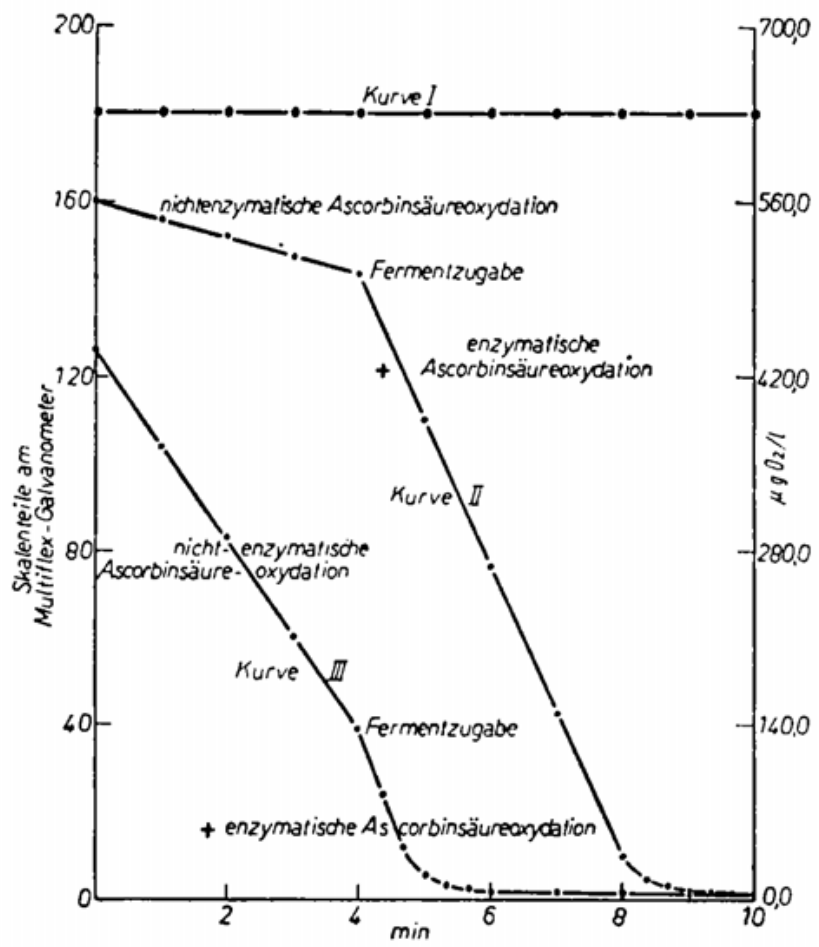

Abbildung H. Verfolgung des Reaktionsverlaufs der nichtenzymatischen und der enzymatischen Ascorbinsäureoxydation durch Kartoffelphenoloxydaselösung

Bereich geringer Sauerstoffgehalte ist praktisch unbegrenzt. Als weiteres Beispiel sei noch der durch Kartoffelphenoloxydase gesteigerte Sauerstoffverbrauch von Ascorbinsäure (Vitamin C) angeführt [6] (Abb. H). Die Messung ist auch in alkoholischen Lösungen und Mischungen von Alkohol und anderen organischen Lösungsmitteln möglich [22]. Außer Sauerstoff läßt sich mit derselben Apparatur unter Verwendung besonderer Elektroden auch der Gehalt an Wasserstoffperoxyd (Katalasewirkung) und gelösten Peroxyden messen, ohne daß der gelöste Sauerstoff die Messungen stört [18].

Das Verfahren gestattet es, den Verlauf sämtlicher Reaktionen zu registrieren, bei denen Sauerstoff entsteht oder verbraucht wird. So wurde die Sauerstoffaufnahme von zweiwertigem Eisen- und Manganhydroxyd in Abhängigkeit von den Versuchsbedingungen, insbesondere vom $\mathrm{P}_{H}$-Wert, elektrochemisch gemessen. Die Ergebnisse zeigen sehr anschaulich, in welcher Weise man in kürzester Zeit mit einem Minimum an experimentellem Aufwand den kinetischen Verlauf solcher Vorgänge erfassen kann [4]. 


\section{Literatur}

[1] K. Damaschke, F. Tödt, D. Burk u. O. Warburg. An electrochemical demonstration of the energy cycle and maximum quantum yield in photosynthesis, Biochim. biophysica Acta 12, 347 (1953).

[2] F. Tödt, R. Lenschau, W. Neudert u. K. Junkmann, Anwendung der elektrochem. O.-Messung zur Beobachtung bakteriologischer Stoffwechselvorgänge, Z. Naturforsch. 9b, 607 (1954).

[3] F. Tödt, K. Damaschke u. L. Rothbühr, Die elektrochemische Messung von SauerstoffUmsätzen bei der Photosynthese, Biochem. Z. 325, $210-222$ (1954).

[4] F. Tödt u. K. Stoklossa, Elektrochemische Messung der Oxydationsgeschwindigkeit von $\mathrm{Fe}(\mathrm{OH})_{2}$ und $\mathrm{Mn}(\mathrm{OH})_{2}$ durch gelösten Sauerstoff, Z. Elektrochem. 58, 354-359 (1954).

[5] F. Tödt, R. Leschber u. H. Tarnow, Betriebskontrolle durch elektrochemische Messung der Atmungsgeschwindigkeit von Hefesuspensionen, Z. Naturforsch. 9 b, 743 (1954).

[6] G. Schenck u. G. Berg, Über die Bestimmung der enzymatischen und nichtenzymatischen Oxydation der Ascorbinsäure mit Hilfe der elektrochemischen Sauerstoffmessung nach F. Tödt, Med. Mschr., H. 10, $667-669$ (1955).

[7] F. Tödt, Anwendung der elektrochemischen Sauerstoffmessung, Angew. Chemie, 9/10, 266 bis 270 (1955).

[8] K. Damaschke, L. Rothbühr u. F. Tödt, Photosynthese unter anaeroben Bedingungen, $\mathrm{Z}$. Naturforsch. 10b, $572-578$ (1955).

[9] K. Damaschke, L. Rothbühr u. F. Tödt, Über die Ausbildung der Giftwirkung verschiedener Gifte aus Chlorella pyrenoidosa im ersten Zeitraum, Z. Naturforsch. 10 b, 215-222 (1955).

[10] K. Damaschke, L. Rothbühru. F. Tödt, Anwendung der elektrochemischen Sauerstoffmeßmethode für Messungen in biologischen Medien und ihre Ausführung, Biochem. Z. 326, 424-432 (1955).

[11] W. Schwarz, Zur elektrochemischen Sauerstoffmessung nach F. Tödt, Werkstoffe u. Korrosion, H. 11, $527-535$ (1955).
[12] F. Tödt, Neue Ergebnisse elektrochemischer Sauerstoffmessungen, DECHEMA-Monographien, 1956.

[13] K. Damaschke u. F. Tödt, Anwendung der elektrochemischen $\mathrm{H}_{2} \mathrm{O}_{2}$-Messung auf die Verfolgung des katalysierten Zerfalls von $\mathrm{H}_{2} \mathrm{O}_{2}$ durch Blutkatalase, Z. Naturforsch. 11 b, H. 11 (1956).

[14] K. Damaschke u. F. Tödt, Messung des an Hämoglobin gebundenen Sauerstoffs auf elektrochemischem Wege, Z. Naturforsch. 11 b, H. 12 (1956).

[15] F. Tödt u. G. Woldt, Über elektrochemische Sauerstoffmessungen in Zuckersäften, Z. Zuckerind. 11 (1956).

[16] M. Rohrlich, F. Tödt u. G. Ziehmann, Elektrochemische Methode zur Bestimmung der Lipoxydaseaktivität in Getreide und Getreide. produkten, Fette, Seifen, Anstrichmittel, Ernährungsindustrie 58 (1956).

[17] F. Tödt u. H.-J. Delavier, Die elektrochemische Messung der Oxydation von Glukose und Fruktose durch gelösten Sauerstoff, Z. Zukkerind. 3 (1957).

[18] K. Damaschke u. F. Tödt, Haltbarkeits kontrolle der Milch durch Messung der Keimzahl und der Katalaseaktivität mittels der elektro. chemischen $\mathrm{O}_{2}$ - und $\mathrm{H}_{2} \mathrm{O}_{2}-\mathrm{MeBmethode.} \mathrm{Milch-}$ wissenschaft 89 (1957).

[19] K. Damaschke, R. Lorenz u. F. Tödt, Über den Einfluß von Zuckerzusätzen auf die Atmungsgeschwindigkeit von Hefesuspensionen. Z. Naturforsch. 12 b, 320 (1957).

[20] F. Tödt u. K. Damaschke, Die Hefeatmung bei sehr geringen $\mathrm{O}_{2}$-Konzentrationen. Z. Naturforsch. 12b, 680 (1957).

[21] F. Tödt u. F. Zimmermann, Elektrochemische Messung der Atmungsgeschwindigkeit von Hefesuspensionen in verschiedenen Pufferlösungen und bei verschiedenen $\mathrm{PH}_{\mathrm{H}}-$ Werten. $\mathrm{Z}$ Zuckerind. 271 (1957).

[22] F. Tödt u. W. Pietrulla, Über die Durchführung elektrochemischer $\mathrm{O}_{2}$-Messungen in alkoholischen Lösungen und organischen Lösungsmittelgemischen. Z. Naturforsch. 12 b, 593(1957). 